\title{
Correlation of markers of autophagy andregional metastasis in colorectal cancer
}

\author{
K. Rachkovsky*, S. Naumov \\ Siberian State Medical University, Tomsk, Russia \\ *e-mail:kirillvr@yandex.ru
}

Key words: colorectal cancer, lymphatic metastasis, autophagy, Beclin-1, mTOR

Motivation and Aim: Colorectal cancer (CRC) is the third most frequently diagnosed cancer in the World, more than one million people are diagnosed colon cancer annually. Despite the fact that colorectal cancer is histological homogeneous, in some cases it is impossible to determine the further prognosis and treatment taking into account the tumor stage and it's differentiation. This problem is due to the fact that each tumor has a unique molecular profile, which is characterized by various genetic and epigenetic changes. Over this issue the approach to the treatment of CRC also based onevaluation of tumor molecular and genetic profile. Such technique allows choosing a more specific treatment and predicting the tumor response to therapy. Considering the fact that colorectal cancer is a genetically heterogeneous tumor, the prognosis and treatmentprimarily depends on molecular pathogenesis that is why researching of autophagy and low molecular weight markers in tumor is very promisingly. To study the correlation of autophagy between lymphatic metastasis in CRC and to evaluate the expression characteristics of the studied proteins in the tissue of the primary tumor.

Materials and Methods: We investigated the surgical material obtained from 100 patients with colorectal cancer (stage T1-4N0-2M0), the mean age of patients being $57.4 \pm 9.1$ years. Treatment before the operation was not carried out. By a standard technique, the tissue of the primary tumor node, as well as all the removed and lymph nodes, was histological examined.Primary tumor node and all removed lymphatic nodes were evaluated by standard histological methods. Tumor stage was established by WHO (2010) classification. All cases which wereordinary adenocarcinoma. We also evaluated lymphatic nodes for presents or absence metastatic lesions. The tissue of the primary tumor was subjected to immunohistochemical examination.We used antibodies to Beclin-1 (Abcam, Anti-beclin 1 antibody, rabbit, polyclonal) and mTOR (SpringBioscience, RabbitAnti-HumanmTORPolyclonalbAntibody, rabbit polyclonal) Expression of the above proteins was assessed using the Histo-score method.

Results: The received data indicate that expression of autophagy proteins Beclin-1 and $\mathrm{m}$-TOR was same positive as presence of lymphatic metastasis as their absence. The results also show that the level of expression Beclin-1 and mTOR is significantly higher in those cases when lymph nodes are affected by metastases (respectively: for Beclin-1 $p=0.004$, for $\mathrm{mTOR}-p=0.004$.) We also established positive correlation between level of expression beclin-1 in cells of adenocarcinoma and amount of effected lymphatic nodes.

Conclusion: In this research we established correlation between the expression of Beclin-1 and mTOR proteins in adenocarcinoma cells with lymphatic metastasis. 